\title{
Minireview
}

Experimentontal
Nephrology

Published online: June 6, 2014

DOI: $\underline{10.1159 / 000363323}$

\section{Heat-Shock Proteins and Acute Ischaemic Kidney Injury}

\author{
Stephen O'Neill ${ }^{a}$ Ewen M. Harrison ${ }^{a}$ James A. Ross ${ }^{b}$ Stephen J. Wigmore ${ }^{a}$ \\ Jeremy Hughes ${ }^{\mathrm{a}}$ \\ ${ }^{a}$ MRC Centre for Inflammation Research and ${ }^{b}$ MRC Centre for Regenerative Medicine, Tissue Injury and Repair \\ Group, University of Edinburgh, Royal Infirmary of Edinburgh, Edinburgh, UK
}

\section{Key Words}

Acute kidney injury · Ischemia-reperfusion injury .

Heat-shock protein

\begin{abstract}
The incidence of acute kidney injury due to ischaemia-reperfusion injury (IRI) is rising but effective treatments and preventative approaches are currently lacking. IRI is also an inevitable consequence of kidney transplantation and significantly contributes to delayed graft function. Heat-shock proteins (Hsps) are highly conserved and ubiquitously expressed molecular chaperones that help maintain and restore normal cellular function in the kidney following IRI. $\mathrm{Hsp} 70$ is one of the most frequently studied Hsps because of potential cytoprotective properties and attractiveness as a therapeutic target. However, the protective properties of Hsp70 in renal IRI are not fully understood and putative modes of protection include correction of protein conformation, cytoskeletal stabilisation, anti-inflammatory effects, requirement in autophagy, anti-apoptotic properties, influence over macrophage phenotype and stimulation of regulatory $T$ cells. Significant clinical interest has been generated about the possibility of applying pharmacological agents to induce $\mathrm{Hsp} 70$ and prevent renal IRI, but prior to this, an increased mechanistic understanding of the protective nature
\end{abstract}

of $\mathrm{Hsp} 70$ is needed. In particular, further investigation of $\mathrm{Hsp}$ expression on inflammatory cell behaviour is required as this could lead to the development of new therapeutic strategies for enhancing recovery following renal IRI and broaden the range of these therapies to a wider group of patients.

C 2014 S. Karger AG, Basel

\section{Acute Ischaemic Kidney Injury}

The incidence of acute kidney injury (AKI) is increasing and represents a significant global health concern [1]. One of the commonest causes for AKI is renal ischaemia, which may result from multiple and often interrelated causes such as hypoperfusion, blood loss and surgery [2]. However, apart from supportive renal replacement therapy, no other therapies exist for patients with AKI and the best treatments presently offered are avoidance of further kidney damage through careful resuscitation, effective treatment of sepsis and avoidance of nephrotoxic medications [3]. In addition, despite anticipated complications, effective preventative strategies prior to predictable renal insults (e.g. elective cardiac surgery) are currently unavailable for patients at high risk of developing postoperative AKI.

\section{KARGER}

E-Mail karger@karger.com

www.karger.com/nee
(C) 2014 S. Karger AG, Basel

$1660-2129 / 14 / 1264-0167 \$ 39.50 / 0$ 
Table 1. Summary of the main well-established functions of the various mammalian Hsp families

\begin{tabular}{llll}
\hline $\begin{array}{l}\text { Mammalian family } \\
\text { Hspation }\end{array}$ & $\begin{array}{l}\text { Principal } \\
\text { locammary of structural features and domains }\end{array}$ & Main established functions \\
\hline Hsp90 & cytosol & $\begin{array}{l}\text { homodimer with two cytosolic isoforms a and } \beta, \\
\text { dimerization occurs at the C-terminal and nucleotide } \\
\text { exchange at the N-terminal }\end{array}$ & $\begin{array}{l}\text { chaperone for a multitude of client proteins } \\
\text { and regulator of protein complex formation }\end{array}$ \\
\hline Hsp70 & $\begin{array}{l}\text { cytosol/ } \\
\text { nucleus/ } \\
\text { mitochondria }\end{array}$ & $\begin{array}{l}\text { consists of a N-terminal (ATPase domain) and a } \\
\text { C-terminal substrate-binding domain connected by }\end{array}$ & $\begin{array}{l}\text { protein trafficking and degradation } \\
\text { refolding of denatured proteins during stress } \\
\text { anti-apoptotic properties }\end{array}$ \\
\hline Hsp60 & mitochondria & $\begin{array}{l}\text { arranged as two stacked heptameric rings with } \\
\text { three domains (apical, intermediate and equatorial) }\end{array}$ & $\begin{array}{l}\text { mitochondrial protein folding and assembly } \\
\text { Hsp40 }\end{array}$ \\
$\begin{array}{llll}\text { cytosol/ } \\
\text { nucleus }\end{array}$ & $\begin{array}{l}\text { J-domain that stimulates the ATPase activity of } \\
\text { Hsp70 and C-terminal that loads polypeptides to }\end{array}$ & $\begin{array}{l}\text { regulates activity of Hsp70 } \\
\text { binds non-native protein } \\
\text { processes pro-collagen }\end{array}$ \\
\hline Hsp27 & cytosol & $\begin{array}{l}\text { conserved C-terminal and highly variable } \\
\text { N-terminal (WDPF domain) }\end{array}$ & preventing unfolded protein aggregation \\
\hline
\end{tabular}

Renal ischaemia-reperfusion injury (IRI) to donor kidneys is also an inevitable consequence of renal transplantation and the resultant organ damage significantly contributes to delayed graft function as well as poorer long-term outcomes [4]. Strategies to protect donor kidneys from ischaemic injury are similarly lacking and are urgently needed to improve both early and late graft function. In this setting, effective treatments could even lead to expansion of the donor pool by improving the outcome for kidneys severely damaged by ischaemia [5]

\section{Heat-Shock Proteins}

Heat-shock proteins (Hsps) are abundant intracellular proteins that are phylogenetically highly conserved. Many Hsps are constitutively expressed and are molecular chaperones, which carefully balance proteostasis through guiding the folding of newly synthesised proteins and regulation of protein translocation. There are several Hsp families each with specific properties that have been well established. Hsps are subdivided into families according to their molecular weight in kilodaltons $(\mathrm{kDa})$. With increasing knowledge, the number of Hsps has greatly expanded resulting in more members being added in the various Hsp families. To enable better classification of Hsps, Kampinga et al. [6] have therefore devised guidelines for the nomenclature of Hsps based on assignments made by the HUGO Gene Nomenclature Committee and used in the National Center of Biotech- nology Information Entrez Gene database for the heatshock genes.

The functions of Hsps include chaperoning of client proteins, regulation of protein complex formation, protein trafficking, refolding of denatured proteins, mitochondrial protein folding and assembly, targeting of misfolded proteins for proteasomal degradation, preventing unfolded protein aggregation and inhibiting apoptosis. To fulfil these diverse roles, each Hsp family has unique structural features and domains (table 1) [6, 7]. However, the multifaceted nature of Hsps is increasingly being recognised and various new roles are being appreciated as the level of understanding increases.

\section{Heat-Shock Response}

Expression of Hsps can be markedly upregulated by various stressors, including heat, in a process termed the heat-shock response. This promotes cellular survival under stressful conditions, as Hsps are also able to assist with numerous reparative processes including the refolding of denatured proteins and removal of irreparably damaged proteins that would otherwise accumulate and initiate cell death [8].

Furthermore, a prior heat-shock response may confer cytoprotection from subsequent injuries such as IRI [9]. This cellular capability fits well with the clinical concept of preconditioning, which is the use of an earlier stress treatment in order to confer later protection from a 
known impending injury. Hsps are therefore prime therapeutic candidates to be used in the context of predictable injury [10]. Indeed, Hsps, and especially Hsp70, have been extensively studied in this context. Nevertheless, despite extensive experimental investigation, reliable manipulation of Hsp responses for clinical application has thus far remained elusive.

\section{Pharmacological Modulation of Hsp Expression}

Hsp70 is one of the most frequently studied Hsps because of its potential anti-inflammatory properties and attractiveness as a therapeutic target due to its wide tissue distribution and ability to interact with a multitude of other molecules [11]. Even though Hsp70 is highly inducible, it may not be clinically desirable to generate Hsp70 by a classical heat-shock response involving cellular stressors. In addition, physical preconditioning approaches often involve an additional thermal or ischaemic injury with potential associated negative consequences [5]. An example is renal ischaemic preconditioning, which is thought to confer some of its proposed protection from renal IRI by induction of Hsps but unfortunately depends on time-consuming and often surgeon-dependent intraoperative manoeuvers to be implemented in a clinical environment [5]. Local ischaemic preconditioning is also not directly applicable to the treatment of the various medical causes of AKI such as hypoperfusion and sepsis. In light of this, approaches to induce Hsp70 without the need for preceding cellular stresses have been sought. As such, recent research in this area has begun to focus on pharmacological induction of Hsp70 for potential therapeutic gain. In the setting of renal IRI, protective Hsp70 overexpression strategies have included the use of reagents such as geranylgeranylacetone, glutamine, radicicol, geldanamycin and geldanamycin analogues [reviewed in 5].

To date, these treatments have been used only in murine models of IRI. The main barrier to the translation of these treatments to clinical use is the lack of mechanistic understanding of how Hsp70 induction results in kidney protection. There is also further uncertainty regarding non-specific mechanisms of protection offered by the various pharmacological therapies used thus far [5]. If these issues could be clarified, then Hsp70-inducing drugs could be developed and further or newer, more highly specific and less toxic medications could be designed.

Heat-Shock Proteins and Kidney Injury

\section{Hsps and Renal IRI}

IRI is a complex event that involves numerous classes of cells and various biological processes such as cell death, microvascular dysfunction, altered transcription and immune activation [12]. Hsps are believed to prevent injury and restore normal cellular function in the kidney following IRI [2]. Indeed, there is a marked increase in renal Hsp expression following renal IRI with Hsp70 gene products showing a 43-fold increase and Hsp27 a 12 -fold increase [13]. The impression that Hsps are cytoprotective following renal IRI is reinforced by evidence from a variety of experimental studies [reviewed in 5]. Investigations involving Hsp70-/- mice further underpin the importance of Hsp70 in the prevention of renal IRI. Hsp70-/- mice have worse kidney function, tubular injury and survival following renal IRI. The protective effect from renal IRI provided by the Hsp70-inducing agent, geranylgeranylacetone, is also abrogated in Hsp70 knockout mice [14]. Although many of the models adopted have focused on the effect of inducing Hsp70, other strategies have been used to manipulate Hsp responses and protect kidneys from ischaemic damage. These include inhibition of Hsp90, which may mediate protection through induction of Hsp70 or NF- $\kappa \mathrm{B}$ deactivation, and selective renal overexpression of Hsp27 [5, 15-17].

Despite this potential for cellular protection strategies involving Hsps, it is often unclear which particular function of the multifunctional Hsp molecule is key to reducing the injury and it has been suggested that there may be a combination of effects including: repair of damaged proteins, prevention of unfolded protein aggregation, targeting of damaged protein for degradation, inhibition of apoptosis, cytoskeleton stabilisation and immunological effects upon leukocytes [7]. It is also increasingly recognised that mononuclear phagocytes and lymphocytes, key cells involved in renal IRI, exhibit remarkable plasticity and are capable of both initiating and resolving tissue injury [18]. This raises the additional possibility that Hsp expression could have an important influence over the behaviour of these cell types as well $[19,20]$.

\section{Potential Cellular and Molecular Mechanisms of Action of Hsp70}

The protective properties of Hsp70 in renal IRI are not fully understood and putative modes of protection include correction of protein conformation, cytoskeletal stabilisation, anti-inflammatory effects, requirement in

Nephron Exp Nephrol 2014;126:167-174 
autophagy, anti-apoptotic properties, influence over macrophage phenotype and stimulation of regulatory $\mathrm{T}$ cells (Tregs) (fig. 1).

\section{Correction of Protein Conformation}

Variations in protein location and abnormal protein folding occur in many kidney diseases and are central components of ischaemic injury [7]. Hsps exhibit complex protective mechanisms that can prevent the formation of non-specific protein aggregates and assist proteins in the acquisition of their native structures [21]. Following cellular injury, Hsps refold damaged proteins, mark irreparably damaged proteins for removal, limit the accumulation of misfolded proteins and aid appropriate folding of nascent proteins [7]. Hsp70 is one of the most highly conserved of these molecular chaperones and its functions encompass regulation of protein transport, prevention of abnormal protein aggregation and resolubilisation of misfolded proteins [10]. Specifically in the injured kidney, Hsps also have a role in the management of protein assembly and stabilisation of multi-protein complexes as well as translocation of proteins to their appropriate intracellular locations [7].

Intracellular Protection and Cytoskeletal Stabilisation

Hsps protect cell structure and facilitate survival under conditions that would normally be lethal. Following kidney injury, Hsps may be capable of sheltering the cell mitochondria from reactive oxygen species, conserving ATP levels, repairing DNA damage, maintaining tight junction integrity and preventing calcium redistribution within the cell [7]. The actin cytoskeleton is also an integral component of the architecture of cells but cellular stresses can lead to major defects [21]. For instance, following renal IRI, renal tubular cells rapidly lose their polarity and the cytoskeleton becomes grossly disrupted. However, in experimental renal IRI models, Hsps (and in particular Hsp70) play a pivotal role in restoring renal tubular cell cellular polarity and repairing essential proteins that are involved in stabilising cytoskeletal structures [reviewed in 10].

\section{Anti-Inflammatory Effects}

It is now recognised that inflammation is perhaps the most critical pathophysiological process involved in the propagation of renal IRI [2]. Hsp70 can limit pro-inflammatory NF- $\kappa B$ signalling in kidney IRI by either stabilising I $\kappa$ B, inhibiting NF- $\kappa$ B p 65 translocation to the nucleus or marking pro-inflammatory Hsp90 client proteins for degradation [5]. Hsps may also exert potent anti-in- flammatory effects. For example, in clinical trials in patients with chronic inflammatory diseases like arthritis, Hsp40 and Hsp60, peptides have been shown to modulate T cell responses by inhibiting division of CD4+ and CD8+ $\mathrm{T}$ cells and promote the production of anti-inflammatory cytokines including interleukin-10, interleukin-6 and transforming growth factor- $\beta[11,22,23]$. This suggests that Hsps may have an important role in preventing or arresting inflammation in renal IRI and thus protecting renal function. It also hints at distinct translational potential for this area of research.

\section{Requirement in Autophagy}

Autophagy is a catabolic process that degrades cytoplasmic components through the action of lysosomes. Autophagy is induced under stress and plays an adaptive role in cell survival. Upregulation of autophagy in renal tubular cells ameliorates both IRI and cisplatin-induced AKI [24]. Hsp70 is required for autophagy due to many forms of stress [25]. In an immune context, Hsp70 is also associated with autophagy, leading to a preferential uploading of Hsp70 peptides in MHC class II molecules of stressed cells. Tregs then target the conserved Hsp70 peptides that may then be presented in these conditions leading to the repression of inflammation [11].

\section{Anti-Apoptotic Effects}

Ischaemic injury leads to both necrosis and apoptosis of renal tubular cells, which results in the release of damage-associated molecular pattern molecules and propagation of inflammation [26]. A stressed but not critically injured renal tubular cell may undergo apoptosis and cell death or alternatively the cell can survive and recover function. This is in contrast to lethally injured cells that inevitably succumb to apoptosis or necrosis and therefore cannot be salvaged. As a consequence, it is the outcome of these stressed but viable cells that would seem the most amenable to cytoprotective interventions [10].

Hsps interact with important proteins involved in apoptotic pathways and this has crucial consequences for cell survival, proliferation and apoptosis following IRI [27]. For instance in renal IRI, Hsp70 limits apoptosis by controlling the activity of the kinases Akt and glycogen synthase kinase $3 \beta$ that regulate the activity of the proapoptotic protein Bax [14]. As a result, renal epithelial cells might be rescued from apoptotic cell death following Hsp induction [10]. It is therefore of interest that cortical Hsp70 levels following renal IRI inversely correlate with apoptosis, tubular injury and renal dysfunction [14]. 


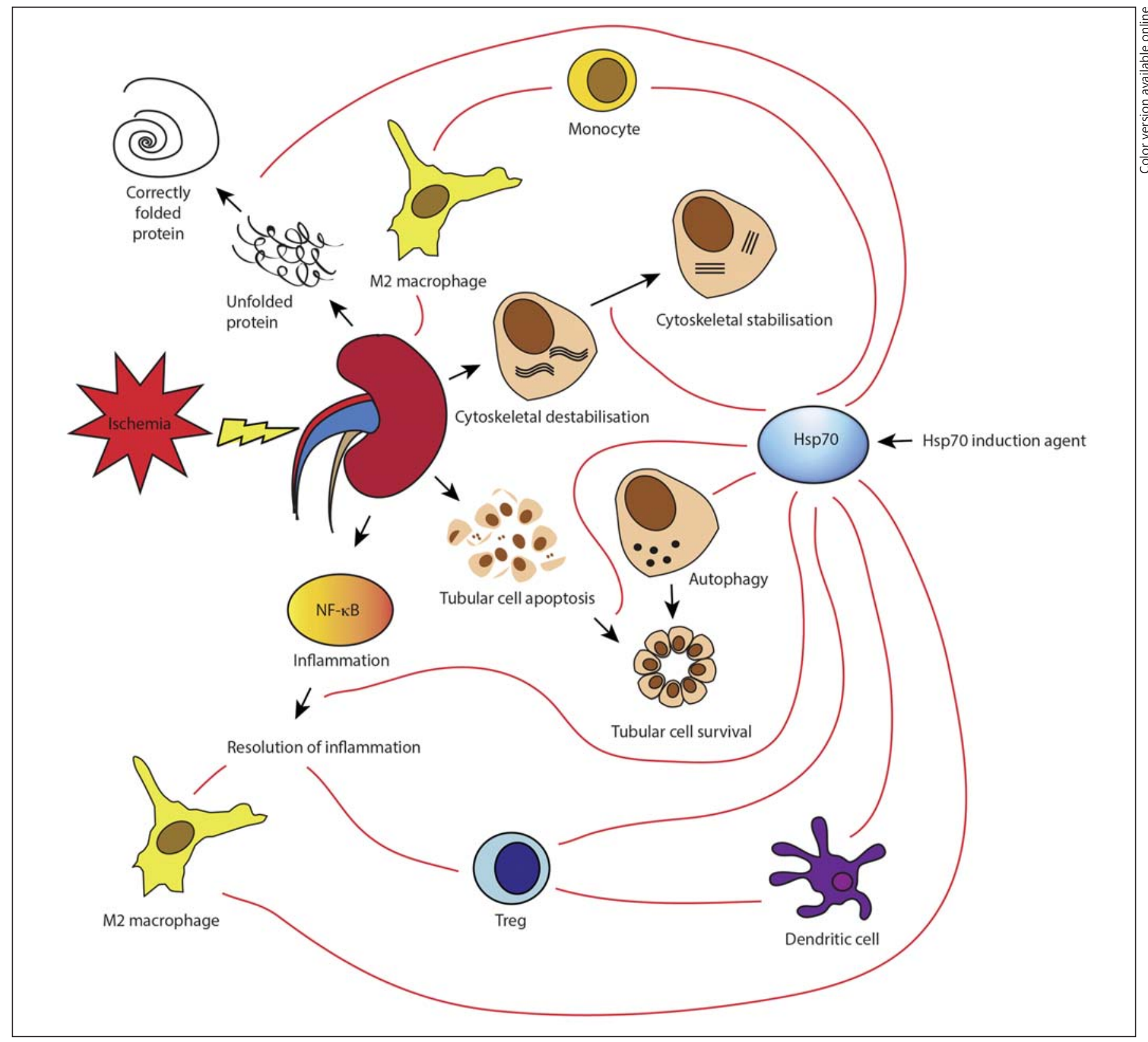

Fig. 1. Potential cellular and molecular mechanisms of action of Hsp70 in renal IRI. Hsp70 induction in IRI may correct cytoskeletal destabilisation, reduce aggregation of unfolded proteins, promote cell survival through autophagy and prevention of apoptosis and resolve inflammation by interrupting inflammatory signalling and modulating cell behaviour.

Influence on the Phenotype of Mononuclear Phagocytes Mononuclear phagocytes are pivotally involved at various stages of renal IRI and depending on the phenotype they exhibit and the time point following injury, they can be viewed as either injurious (M1 macrophages) or reparative (M2 macrophages). Hsps appear capable of pro- moting an anti-inflammatory phenotype in monocytes, which leads to release of anti-inflammatory cytokines such as interleukin-10 and suppression of harmful cytokines including interferon- $\gamma$ [11]. Hsps may also influence macrophage phenotype with transcriptomic profiling during the differentiation and polarisation of human 
macrophages showing distinct patterns of Hsp expression. For example, upon activation to a M1 phenotype a significant upregulation of members of the Hsp70 family (HspA2 and HspA8) and Hsp90 family (Hsp90AA1) are observed, which suggests their influence over the effector stage of macrophage activation. While in M2 macrophages other members of the Hsp70 (HspA13), Hsp40 (DNAJB5) and Hsp27 (HspBAP1) families are significantly upregulated [28].

In experimental renal IRI models, preconditioning with heat protects rat kidneys from ischaemic damage by inducing $\mathrm{Hsp} 70$ and suppressing NF- $\kappa \mathrm{B}$ activation. Heat preconditioning also reduces chemokine (C-C motif) ligand 2 (CCL2) expression, which is necessary to attract monocytes to the site of injury [29]. Furthermore, Hsp70 induction by either geranylgeranylacetone or repetitive hypoxic preconditioning reduces macrophage infiltration, protects kidney function, attenuates tubular damage and reduces renal tubular cell apoptosis following renal IRI $[30,31]$. The causality of Hsp70 in these observations has been asserted in subsequent investigations that showed reversal of this effect following the addition of the Hsp70 inhibitor, quercetin [30]. However, despite being widely known, quercetin is a non-specific inhibitor of Hsp70 and further investigation of Hsp70 in this context is needed [5].

\section{Induction of Regulatory $T$ Cells}

Tregs are a critical subtype of lymphocytes that display potent immunosuppressive effects and act to coordinate immunological responses. In renal IRI, infiltration of Tregs into the kidney has previously been shown to ameliorate injury and manipulation of Treg activity could represent a novel therapeutic approach for treating ischaemic AKI [18].

Recently, it has been demonstrated that the protective effect of Hsp70 in renal IRI is Treg-dependent with the influence of Hsp70 on Treg behaviour possibly being driven through splenic CD11c+ dendritic cells $[19,20]$. Kim et al. [19] used Treg depletion strategies to show that heat preconditioning induced Hsp70 and mediated protection of kidney function from renal IRI that was Tregdependent. Further experiments involving the administration of Hsp70 inhibitors and as well as adoptive transfer of T cells from heat-preconditioned Hsp70-/- mice suggested that Hsp70 was important for generating protective Tregs. In the latter experiments it was shown that following renal IRI, nude mice that previously exhibited reduced kidney injury had injury reconstituted by the adoptive transfer of $\mathrm{T}$ cells from wild-type mice. In con- trast, the transfer of $\mathrm{T}$ cells from heat-preconditioned wild-type mice to nude mice did not reconstitute kidney injury. However, nude mice given $\mathrm{T}$ cells from heat-preconditioned Hsp70-/- mice exhibited partial reconstitution of kidney injury.

It has also been suggested elsewhere in other disease processes that Hsp70 is capable of inducing tolerogenic dendritic cells that can go on to stimulate protective Treg functions [11]. In fact, for certain chronic inflammatory conditions such as diabetes and arthritis, the concept of Hsps acting as immunological peptides and thus potential targets for $\mathrm{T}$ cell modulation as well as anti-inflammatory Treg responses has already been established in human studies [23].

\section{Negative Effects of Hsp70 Induction}

In addition to potent anti-inflammatory effects, Hsp70 may potentially exert pro-inflammatory effects. A theory exists that Hsp70 located in the cytosol reduces inflammatory signalling, however Hsp70 released into extracellular compartments displays immune-stimulatory or 'chaperokine' properties that are similar to those exhibited by damage-associated molecular pattern molecules. The cellular compartment that Hsp70 resides in may be influenced by the extent of tissue injury and could be independent of pharmacological induction [5]. This area remains poorly understood and a recent review has highlighted the need for further studies aimed at more accurately characterising Hsp70 and its overall effect on the inflammatory equilibrium [11].

\section{Future Prospects for Translation to Patients}

\section{Preconditioning and Prevention of IRI}

In the context of an impending ischaemic insult, the aim is to administer an agent capable of increasing the level of Hsp70 in the kidney prior to the onset of IRI. Reagents such as geranylgeranylacetone, glutamine, radicicol, geldanamycin and geldanamycin analogues have been used in this context but to date only in an experimental setting [reviewed in 5]. This would prime protective cellular mechanisms and prevent kidney damage from being inflicted. For instance, patients awaiting cardiac or vascular surgeries that are predicted to be at high risk of developing postoperative AKI may benefit from Hsp70 induction prior to surgery. High-risk patients (e.g. pre-existing $\mathrm{CKD}$, diabetes, extensive vascular disease, 
etc.) awaiting toxic drug or radiological contrast exposure may also benefit from such preventative measures [10]. This type of preconditioning strategy clearly has much potential because it would allow prevention of IRI at the earliest stage. Despite this there may be difficulty deciding who should receive such treatments but the development of various predictive models of outcome following diverse interventions may well be able to stratify patients according to the risk of developing AKI in the future.

In transplantation, delivering an agent to the organ donor prior to organ procurement could also induce Hsp70 in the kidney and this could facilitate protection from the inevitable insult of IRI. In addition, prior to implantation it may be feasible to treat a kidney ex vivo with an Hsp70-inducing agent administered by machine perfusion [5]. However, this may only be applicable to normothermic perfusion strategies since the activity of certain pharmacological agents may be decreased at the lower temperatures used for hypothermic machine perfusion.

\section{Enhancing Recovery following IRI}

Despite evidence that Hsp70 induction can mediate renal protection after IRI [14], current research in this area has focused almost exclusively on preconditioning and to a lesser extent on deploying protective Hsp expression strategies in the recovery from renal IRI. However, further investigation of recovery therapy might increase the capacity of these treatments to help patients that have already suffered ischaemic AKI in a native or transplanted kidney. Moreover, treatment of the transplant recipient may be more translationally feasible as there are ongoing ethical and logistical barriers to the pretreatment of donors.

It is anticipated that a greater emphasis on recovery phase therapy may evolve from further understanding of how Hsp70 induction may interact with specific inflammatory cell types in renal IRI. For instance, confirmation that Hsp70 induction influences circulating or infiltrating inflammatory cells either involved in the propagation or recovery from IRI would provide stronger evidence that treatment in the recovery stage could also be of benefit.

Following transplantation the recipient's circulation continues to carry infiltrating inflammatory cells to the kidney. These circulating inflammatory cells are possible treatment targets due to their ability to either sustain or resolve tissue inflammation [18]. Hsp70 induction may occur in immune cells remote from the kidney following heat shock and plays an important role in mediating protective Treg responses in renal IRI [19, 20]. Consequent- ly, it is predicted that an Hsp70-inducing candidate drug could also modulate the immune activity of Tregs and other infiltrating inflammatory cells through a similar systemic effect. There is thus potential for using Hsp70inducing treatments in recipients prior to transplantation or even after the kidney has been implanted.

However, these effects have not been unequivocally confirmed as yet and Hsp-inducing agents may still have their major impact on renal tubular cells or renal resident leukocytes. As such, Hsp70 induction within the kidney parenchyma itself would be of prime importance with circulating or infiltrating inflammatory cell populations not representing the key target. In this case, researchers will need to induce protective levels of Hsp70 within renal tubular cells or renal resident inflammatory cells at the earliest possible stage. The focus will therefore remain on preconditioning the kidney, which may slow translational progress in this field particularly in the context of transplantation.

\section{Conclusions}

The possibility of applying pharmacological agents to induce Hsp70 and prevent renal IRI has significant potential to benefit patients at high risk of AKI or undergoing renal transplantation. However, prior to converting these therapies into a clinical context, an increased mechanistic knowledge of the protection offered by Hsp70 is required. From a translational standpoint, an enhanced understanding of the inflammatory cell types involved could also broaden the scope of these therapies to wider group of patients.

\section{Acknowledgements}

S. O’Neill has been supported by a Maurice Wohl Research Fellowship from the Royal College of Surgeons Edinburgh, a Mason Medical Research Trust Fellowship, and a Medical Research Council Clinical Research Training Fellowship.

\section{Disclosure Statement}

The authors have no conflicts of interest to disclose 


\section{References}

$>1$ Lameire NH, Bagga A, Cruz D, De Maeseneer J, Endre Z, Kellum JA, et al: Acute kidney injury: an increasing global concern. Lancet 2013;382:170-179.

$>2$ Sharfuddin AA, Molitoris BA: Pathophysiology of ischemic acute kidney injury. Nat Rev Nephrol 2011;7:189-200.

$\checkmark 3$ Murugan R, Kellum JA: Acute kidney injury: what's the prognosis? Nat Rev Nephrol 2011; 7:209-217.

4 Yarlagadda SG, Coca SG, Formica RN Jr, Poggio ED, Parikh CR: Association between delayed graft function and allograft and patient survival: a systematic review and meta-analysis. Nephrol Dial Transplant 2009;24:10391047.

$\checkmark 5$ O’Neill S, Ross JA, Wigmore SJ, Harrison EM: The role of heat-shock protein 90 in modulating ischemia-reperfusion injury in the kidney. Expert Opin Investig Drugs 2012;21:15351548.

6 Kampinga HH, Hageman J, Vos MJ, Kubota $\mathrm{H}$, Tanguay RM, Bruford EA, et al: Guidelines for the nomenclature of the human heatshock proteins. Cell Stress Chaperones 2009; 14:105-111.

$>7$ Kelly KJ: Heat-shock (stress response) proteins and renal ischemia/reperfusion injury. Contrib Nephrol. Basel, Karger, 2005, vol 148, pp 86-106.

$>8$ Kampinga HH, Craig EA: The HSP70 chaperone machinery: J proteins as drivers of functional specificity. Nat Rev Mol Cell Biol 2010; 11:579-592.

$>9$ Jones Q, Voegeli TS, Li G, Chen Y, Currie RW: Heat-shock proteins protect against ischemia and inflammation through multiple mechanisms. Inflamm Allergy Drug Targets 2011;10:247-259.

10 Aufricht C: Heat-shock protein 70: molecular supertool? Pediatr Nephrol 2005;20:707-713.

$>11$ Borges TJ, Wieten L, van Herwijnen MJ, Broere F, van der Zee R, Bonorino C, et al: The anti-inflammatory mechanisms of Hsp70. Front Immunol 2012;3:95.

$>12$ Eltzschig HK, Eckle T: Ischemia and reperfusion - from mechanism to translation. Nat Med 2011;17:1391-1401.
13 Zhang PL, Lun M, Schworer CM, Blasick TM, Masker KK, Jones JB, et al: Heat-shock protein expression is highly sensitive to ischemiareperfusion injury in rat kidneys. Ann Clin Lab Sci 2008;38:57-64.

14 Wang Z, Gall JM, Bonegio RG, Havasi A, Hunt CR, Sherman MY, et al: Induction of heat-shock protein 70 inhibits ischemic renal injury. Kidney Int 2011;79:861-870.

15 Sonoda H, Prachasilchai W, Kondo H, Yokota-Ikeda N, Oshikawa S, Ito K, et al: The protective effect of radicicol against renal ischemia-reperfusion injury in mice. J Pharmacol Sci 2010;112:242-246.

16 Kim M, Park SW, Chen SW, Gerthoffer WT, D'Agati VD, Lee HT: Selective renal overexpression of human heat-shock protein 27 reduces renal ischemia-reperfusion injury in mice. Am J Physiol Renal Physiol 2010; 299:F347-F358.

17 Harrison EM, Sharpe E, Bellamy CO, McNally SJ, Devey L, Garden OJ, et al: Heat-shock protein 90-binding agents protect renal cells from oxidative stress and reduce kidney ischemia-reperfusion injury. Am J Physiol Renal Physiol 2008;295:F397-F405.

8 Bonventre JV, Yang L: Cellular pathophysiology of ischemic acute kidney injury. J Clin Invest 2011;121:4210-4221.

19 Kim MG, Jung Cho E, Won Lee J, Sook Ko Y, Young Lee H, Jo SK, et al: The heat-shock protein-70-induced renoprotective effect is partially mediated by CD4+CD25+Foxp3+ regulatory $\mathrm{T}$ cells in ischemia/reperfusion-induced acute kidney injury. Kidney Int 2014; 85:62-71.

20 O’Neill S, Hughes J: Heat-shock protein-70 and regulatory $\mathrm{T}$-cell-mediated protection from ischemic injury. Kidney Int 2014;85:57.

21 Richter K, Haslbeck M, Buchner J: The heatshock response: life on the verge of death. Mol Cell 2010;40:253-266.

22 Van Eden W, van Herwijnen M, Wagenaar J, van Kooten P, Broere F, van der Zee R: Stress proteins are used by the immune system for cognate interactions with anti-inflammatory regulatory T cells. FEBS Lett 2013;587:19511958.
23 Keijzer C, Wieten L, van Herwijnen M, van der Zee R, Van Eden W, Broere F: Heat-shock proteins are therapeutic targets in autoimmune diseases and other chronic inflammatory conditions. Expert Opin Ther Targets 2012;16:849-857.

24 Kaushal GP: Autophagy protects proximal tubular cells from injury and apoptosis. Kidney Int 2012;82:1250-1253.

25 Yang Y, Fiskus W, Yong B, Atadja P, Takahashi Y, Pandita TK, et al: Acetylated hsp70 and KAP1-mediated Vps34 SUMOylation is required for autophagosome creation in autophagy. Proc Natl Acad Sci USA 2013;110: 6841-6846.

26 Eleftheriadis T, Lawson BR: Toll-like receptors and kidney diseases. Inflamm Allergy Drug Targets 2009;8:191-201.

27 Lanneau D, Brunet M, Frisan E, Solary E, Fontenay M, Garrido C: Heat-shock proteins: essential proteins for apoptosis regulation. J Cell Mol Med 2008;12:743-761.

28 Fagone P, Di Rosa M, Palumbo M, De Gregorio C, Nicoletti F, Malaguarnera L: Modulation of heat-shock proteins during macrophage differentiation. Inflamm Res 2012;61: 1131-1139.

29 Jo SK, Ko GJ, Boo CS, Cho WY, Kim HK: Heat preconditioning attenuates renal injury in ischemic ARF in rats: role of heat-shock protein 70 on NF- $\kappa \mathrm{B}$-mediated inflammation and on tubular cell injury. J Am Soc Nephrol 2006;17:3082-3092.

30 Suzuki S, Maruyama S, Sato W, Morita Y, Sato F, Miki Y, et al: Geranylgeranylacetone ameliorates ischemic acute renal failure via induction of Hsp70. Kidney Int 2005;67:22102220.

31 Yeh CH, Hsu SP, Yang CC, Chien CT, Wang NP: Hypoxic preconditioning reinforces HIF-a-dependent HSP70 signaling to reduce ischemic renal failure-induced renal tubular apoptosis and autophagy. Life Sci 2010;86: 115-123. 